\title{
SUSY Searches (ATLAS/CMS): the Lady Vanishes
}

\author{
Andy Parker* \\ On behalf of the ATLAS and CMS Collaborations \\ Cavendish Laboratory, University of Cambridge \\ E-mail: parker@hep.phy.cam.ac.uk
}

\begin{abstract}
Recent LHC results from ATLAS and CMS on searches for supersymmetric particles are presented, including the first searches with the $8 \mathrm{TeV}$ centre of mass energy data, from CMS. No indication has yet been seen for any SUSY signal, with the data compatible with the estimated standard model backgrounds. Limits have therefore been set on the masses of various supersymmetric particles, and these are reviewed. Scenarios in which light supersymmetric particles are present, and remain undetected, are also discussed.
\end{abstract}

36th International Conference on High Energy Physics,

July 4-11, 2012

Melbourne, Australia

${ }^{*}$ Speaker. 


\section{Introduction}

Supersymmetry (SUSY) $[1,2,3,4,5,6,7,8,9]$ is the most studied extension to the Standard Model (SM). The literature contains many scenarios, often modelled in great detail, which make predictions that SUSY particles should be observable at the LHC, with masses in the region of $\sim 1 \mathrm{TeV}$, and these predictions have been tested by many analyses. At this conference alone, over 30 relevant talks were given by ATLAS [10] and CMS [11] in the parallel sessions, with some talks describing five separate analyses. This review cannot hope to cover the wealth of new information available, or even to provide references to every relevant analysis. Instead, it will focus on some key issues and a few of the most decisive results. Readers are encouraged to refer to the proceedings of the parallel sessions for a complete overview of the available results.

The absence of any signal from SUSY so far places high energy physics in a similar situation to the heroine of the movie The Lady Vanishes (Hitchock, 1938). She is convinced that she boarded her train in the company of a mysterious lady. But no trace of her can be found, and her presence is denied by all the passengers. Our heroine wonders whether the lady is a figment of her imagination; hidden somewhere on the train; or, perhaps, dead. In this review we will ask similar questions: why should we believe in the existence of SUSY; is SUSY hiding somewhere in our existing data; should SUSY be considered to be an interesting, but dead, hypothesis?

There are many reasons to believe that SUSY might be relevant to physics in the TeV energy range. We already have evidence of physics beyond the SM, from observations consistent with dark matter. R-parity conserving (RPC) SUSY $[12,13]$ offers a heavy weakly interacting particle which is a natural dark matter candidate. We also now have a Higgs-like boson, at a mass close to $125 \mathrm{GeV}$, and the stability of its mass can be protected from quantum loop effects by SUSY partners $[14,15,16,17,18,19]$. The theoretical motivations for proposing SUSY models remain as strong as ever, and so it would be unwise to decide that SUSY is a figment of our imagination, before the searches are complete.

The sensitivity of the current searches depends strongly on which processes are most accessible. The highest reach is achieved for gluinos and for first and second generation squarks, while, for example, stops and charginos have lower cross-sections and are harder to detect, and the search limits are less stringent.

\section{Direct searches for strong production of SUSY states}

The most powerful of the existing searches are based on all-hadronic final states with missing transverse energy $\left(E_{T}^{\text {miss }}\right)$. These target strong production of squarks and gluinos in RPC models, where the emission of two of the lightest SUSY particles (LSPs), normally neutralinos, is responsible for the $E_{T}^{\text {miss }}$. ATLAS has presented a search for all hadronic final states with 2 to 6 jets, and $E_{T}^{\text {miss }}$ [20]. A set of signal regions is defined, based on the jet multiplicity and the transverse momentum $\left(p_{T}\right)$ of the jets, with the final selection made using $m_{\text {eff }}$, the scalar sum of the missing energy and selected jets. Figure 1 shows the $m_{\text {eff }}$ distribution from one signal region, and the final exclusion limit which combines the data from all the signal regions. In order to minimise model dependence, this limit is made in a simplified scenario, in which only squarks and gluinos 

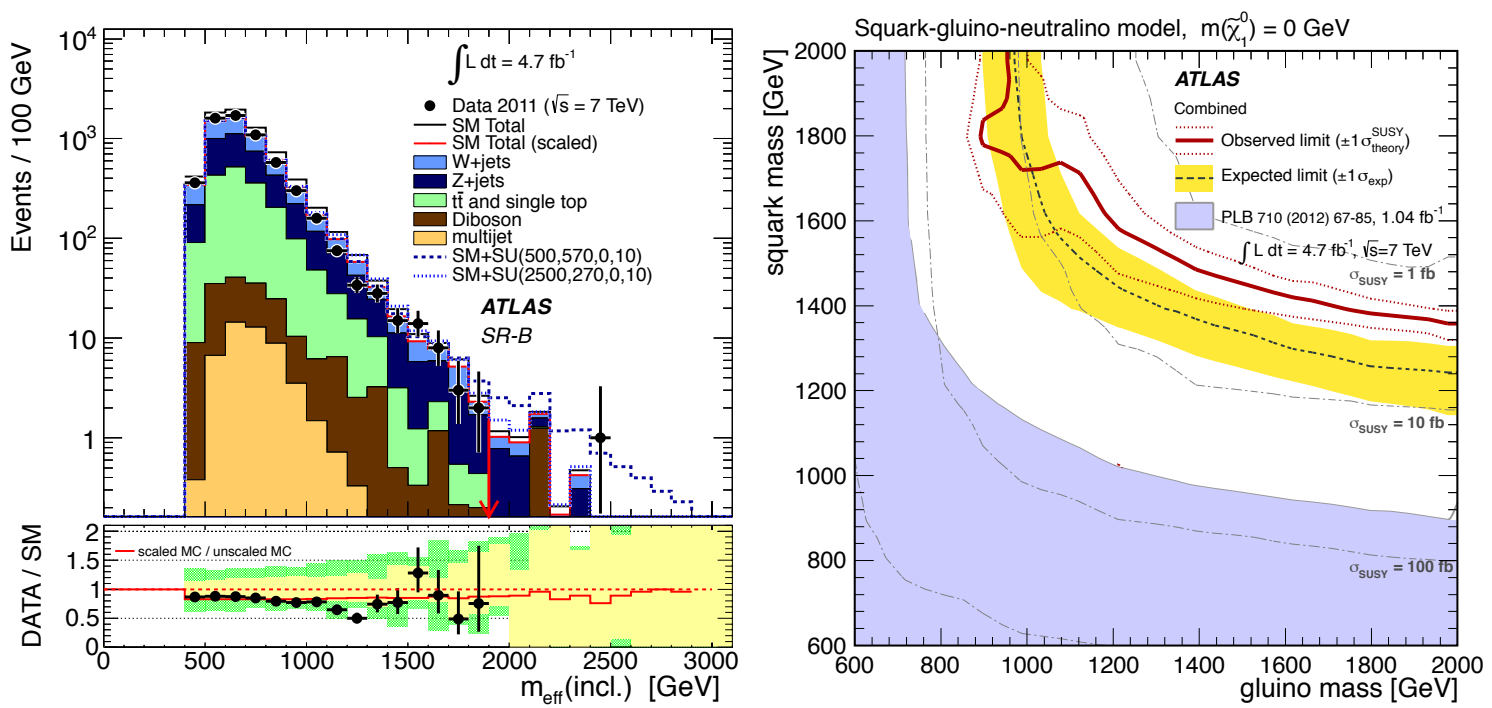

Figure 1: Left: observed $m_{\text {eff(incl.) }}$ distribution for channel B in the ATLAS 0-lepton search [20]. In the top panel, the histograms show the SM background expectations, both before (black open histogram) and after (medium (red) open histogram) use of a fit to scale the expectations to control region observations. Two MSUGRA/CMSSM benchmark model points with $m_{0}=500 \mathrm{GeV}, m_{1 / 2}=570 \mathrm{GeV}, A_{0}=0, \tan \beta=$ 10 and $\mu>0$ and with $m_{0}=2500 \mathrm{GeV}, m_{1 / 2}=270 \mathrm{GeV}, A_{0}=0, \tan \beta=10$ and $m u>0$, illustrating different topologies, are also shown. The arrow indicates the locations of the lower edge of the signal region. The bottom panel shows the fractional deviation of the data from the total unscaled background estimate (black points), together with the fractional deviation of the total scaled background estimate from the total unscaled background estimate (medium (red) line). The light (yellow) band shows the combined experimental uncertainties on the unscaled background estimates from jet energy scale, jet energy resolution, the effect of pile-up, the treatment of energy outside of reconstructed jets and MC simulation sample size. The medium (green) band includes also the total theoretical uncertainties. Right: the $95 \% C L_{s}$ exclusion limits on the $\left(m_{\text {gluino }}, m_{\text {squark }}\right)$-plane in a simplified MSSM scenario with only strong production of gluinos and first- and second-generation squarks, with direct decays to jets and neutralinos. Exclusion limits are obtained by using the signal region with the best expected sensitivity at each point. The black dashed lines show the expected limits, with the light (yellow) bands indicating the 1 sigma excursions due to experimental uncertainties. Observed limits are indicated by medium (maroon) curves, where the solid contour represents the nominal limit, and the dotted lines are obtained by varying the cross section by the theoretical scale and PDF uncertainties. Previous results from ATLAS are represented by the shaded region (blue) at bottom left.

are kinematically accessible, and the LSP mass is set to zero. Because of the lower production cross-section for stops, the limits only apply to first and second generation squarks.

Figure 2 shows data from the equivalent all hadronic search from CMS [21]. This search requires $\geq 3$ jets, and uses the variables $H_{T}=\Sigma p_{T}^{j e t}$ and missing $H_{T}=-\Sigma \vec{p}_{T}^{j e t}$ as discriminants. The data show good agreement with the SM, and Figure 3 shows the resulting exclusion regions in a simplified model with only squark and gluino production. The limits are plotted as a function of the LSP mass, and show that there is no exclusion for LSP masses $>400 \mathrm{GeV}$, due to the falling acceptance, as the LSPs carry away an increasing fraction of the energy.

CMS also presented the first results at $\sqrt{s}=8 \mathrm{TeV}$ at this conference. Figure 4, from [22], shows one exclusion limit from an interesting analysis in which the data are binned according to 


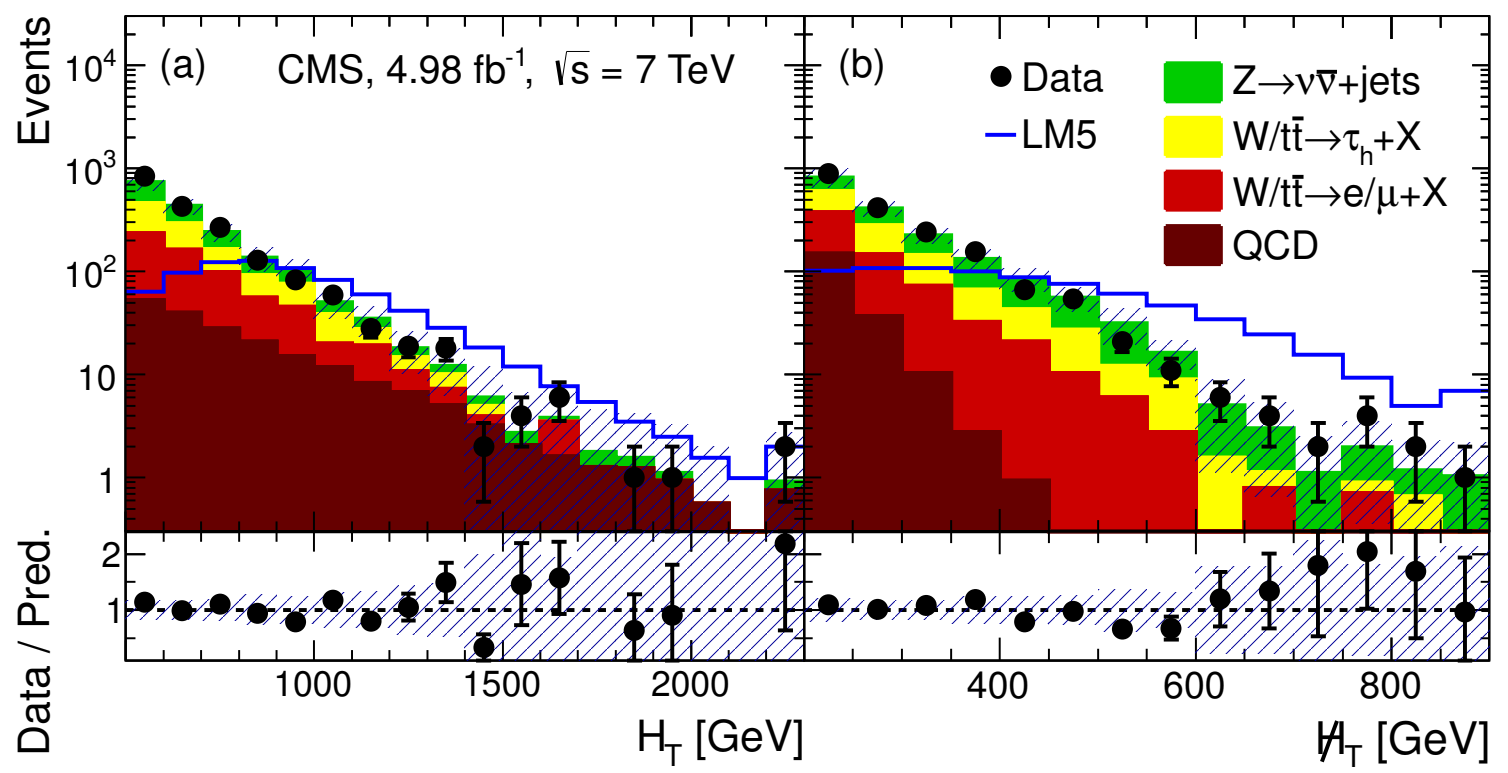

Figure 2: The (a) $H_{T}$ and (b) missing $H_{T}$ distributions in the search data samples (circles) compared with histograms showing predictions of the SM background and a low mass SUSY signal, for events passing the baseline selection, from [21]. The hatched region indicates the uncertainties on the background predictions. The last bin contains all events above the maximum value of $H_{T}$ and missing $H_{T}$ in the figures. The ratio of the observed data to the background predictions is also shown.

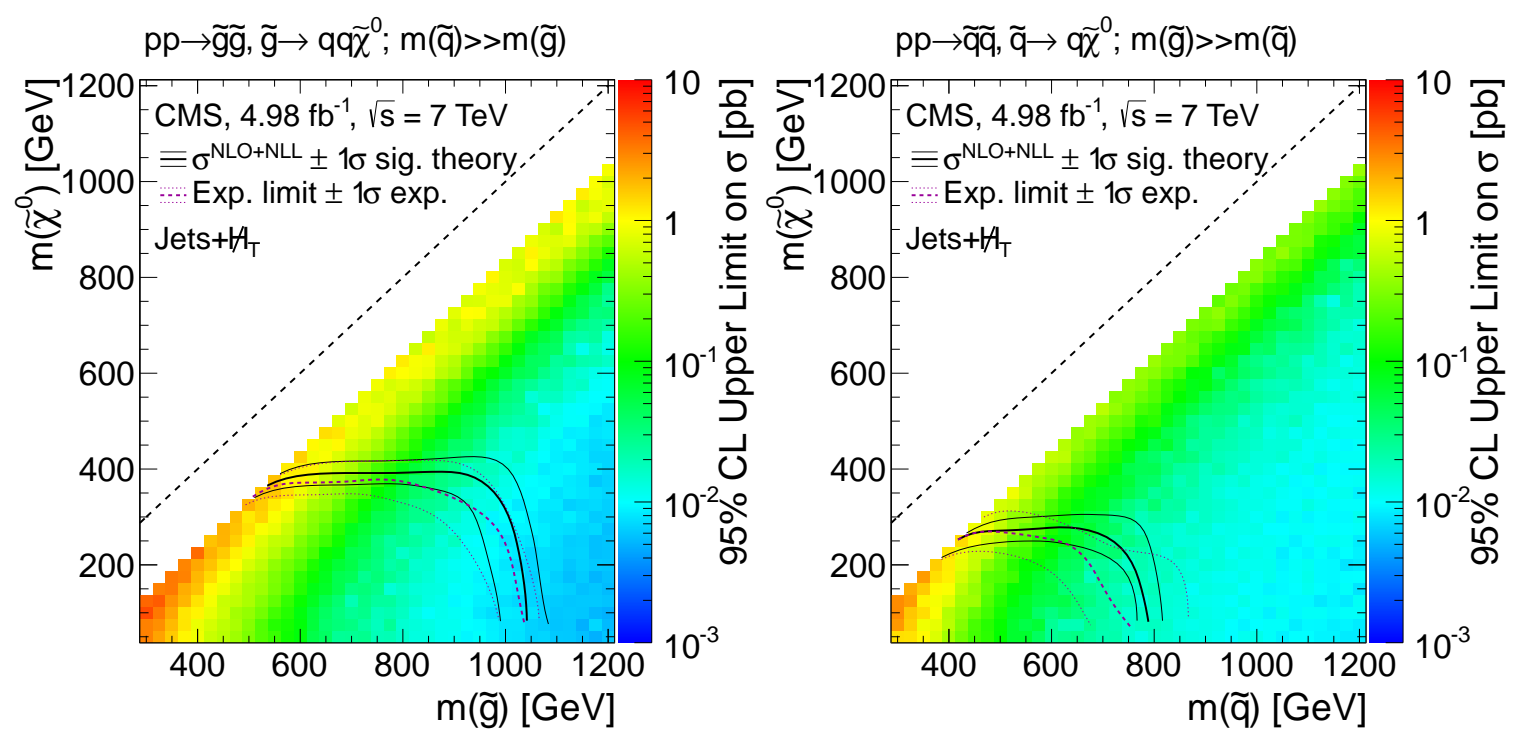

Figure 3: The observed and expected 95\% CL upper limits on the (a) $\tilde{g} \tilde{g}$ and (b) $\tilde{q} \tilde{q}$ cross sections in the (gluino, neutralino) and (squark, neutralino) mass planes obtained with the simplified model [21]. Also shown are the $\pm 1 \sigma$ variation in the expected limit and the variation in the observed limit when the signal cross section is varied by its theoretical uncertainties. 


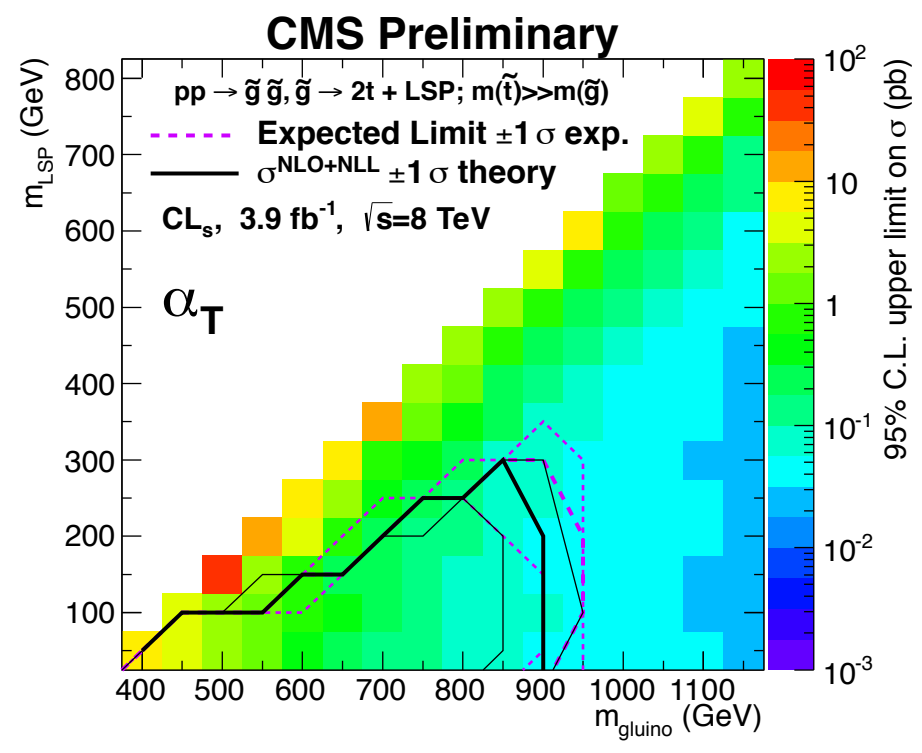

Figure 4: Exclusion cross-section versus model spectrum for pair-produced gluinos, each decaying to a top quark-antiquark pair and a neutralino LSP [22]. The colour scale indicates the observed cross section upper limit (95\% C.L.) for each model point, as a function of the mass of the produced particle and the LSP mass. The solid black line indicates the observed exclusion region assuming nominal NLO SUSY production cross section. The dashed black line indicates the median expected exclusion region, with the dotted (-1sigma) and dotted-dashed (+1sigma) black lines indicating the expected exclusion regions with experimental uncertainties.

the number of $b$-tagged jets. Multi-jet background is rejected using a variable $\alpha_{T}=\frac{E_{T}^{j_{2}}}{M_{T}}$, where $M_{T}^{2}=\left(E_{T}^{j_{1}}+E_{T}^{j_{2}}\right)^{2}-\left(\vec{p}_{T}^{j_{1}}-\vec{p}_{T}^{j_{2}}\right)^{2}$ and $j_{1}, j_{2}$ are the leading jets. $\alpha_{T}=0.5$ for well balanced di-jet events, but can exceed this value for jet systems with missing energy. The analysis produces a very generic search. The results do not yet compete with the full dataset from 2011, but will clearly rapidly exceed the reach shown here as the data sample grows.

\section{Direct searches for weak production of SUSY states}

If the squark and gluino masses are relatively high, the first sign of SUSY might come from weak production processes, and CMS and ATLAS have both presented results in this area (see for example [23, 24]) . Figure 5, from [24], shows exclusion limits from ATLAS on chargino and slepton production. These limits depend on assumptions about the mass hierarchy of the SUSY particles, which also makes it difficult to access the kinematic region where the chargino and neutralino masses are degenerate. The possibility of a very light LSP is therefore not excluded by these searches.

\section{Third generation searches}

The argument that SUSY cancels the divergences in the Higgs mass requires the SUSY particles be close to the $\mathrm{TeV}$ scale to avoid large fine-tuning of the theory parameters. In models like 

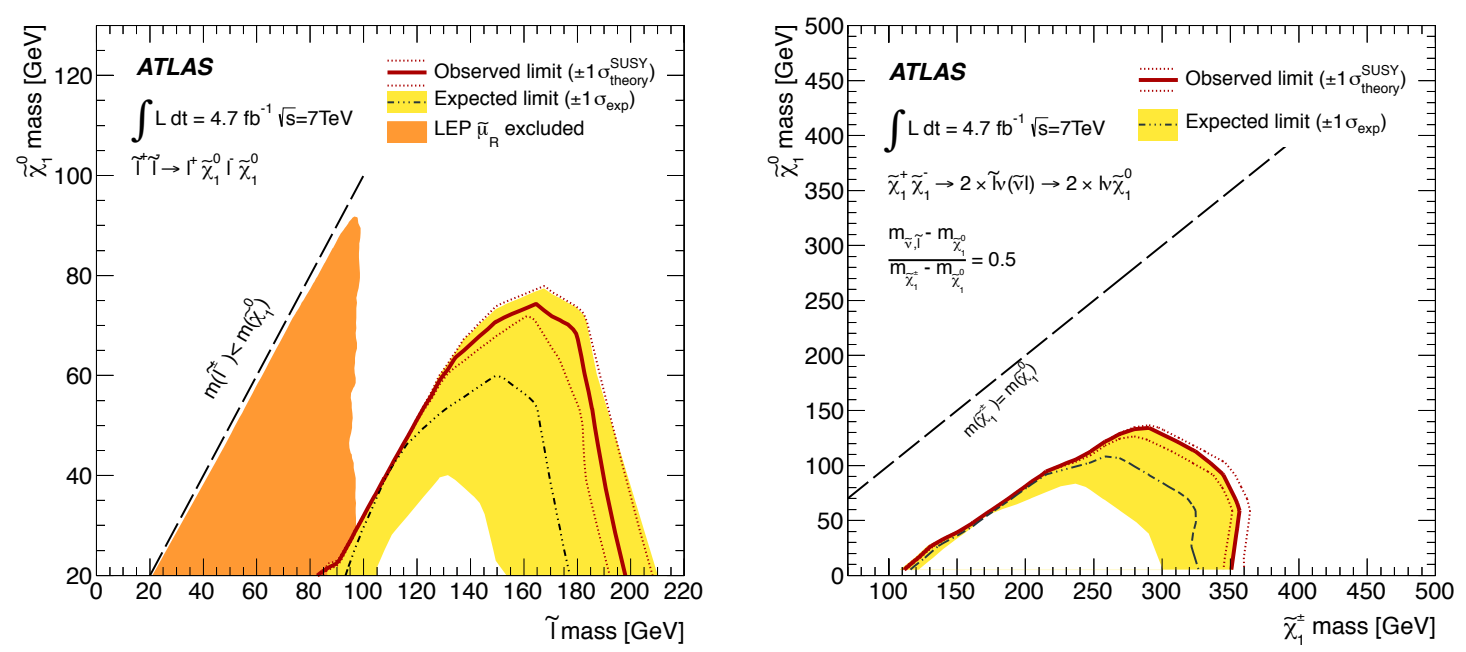

Figure 5: Left: 95\% CL exclusion limits for slepton pair production in the slepton-neutralino mass plane of the direct slepton pMSSM. Right: the same, for charginos [24]. The dashed and solid lines show the 95\% CLs expected and observed limits, respectively, including all uncertainties except for the theoretical signal cross-section uncertainty (PDF and scale). The solid band around the expected limit shows the $\pm 1 \sigma$ result where all uncertainties, except those on the signal cross-sections, are considered. The $\pm 1 \sigma$ lines around the observed limit represent the results obtained when moving the nominal signal cross-section up or down by the $\pm 1 \sigma$ theoretical uncertainty. Illustrated also is the LEP limit [25] on the mass of the right-handed smuon. The LEP limit is a conservative limit on slepton pair production: if right-handed slepton masses are excluded, left-handed sleptons of equivalent masses are automatically excluded.

the Minimal Supersymmetric Standard Model (MSSM) [26, 27, 28, 29, 14], theoretical constraints are imposed on the SUSY spectrum, leading to squark and gluino masses which conflict with the current search limits. However, the dominant term in the Higgs mass correction comes from the top quark loop. Therefore, it is only necessary for the stop to be in the TeV range in order to stabilise the Higgs mass [30, 31]. Other SUSY masses could be in the multi-TeV range and hence beyond current reach. The lower cross-section for third generation squarks would explain why no evidence for strong production has yet been seen.

Searches for third generation SUSY particles are therefore now a priority. CMS presented the first results from the $8 \mathrm{TeV}$ data [32], with a search for same-sign leptons in events with $\geq 2 \mathrm{~b}$ tagged jets. Figure 6 shows two examples of relevant production mechanisms, involving direct pair production of squarks, and production mediated by gluinos. One example of the results is shown in Figure 7, with exclusion limits on the stop mass reaching almost $700 \mathrm{GeV}$ in parts of parameter space. CMS also presented an search for stau production [33], which might be expected in these scenarios.

Because of the variety of production mechanisms and decay modes for stop, these searches are performed in a variety of channels. Figure 8 summarises the results from five ATLAS analyses $[34,35,36,37,38]$. The exclusion region on the right-hand side of the plot comes from direct stop decay to a top quark and neutralino. The regions on the left-hand side rely on decays via charginos, and therefore require assumptions about the mass hierarchy. Two cases are shown: a 

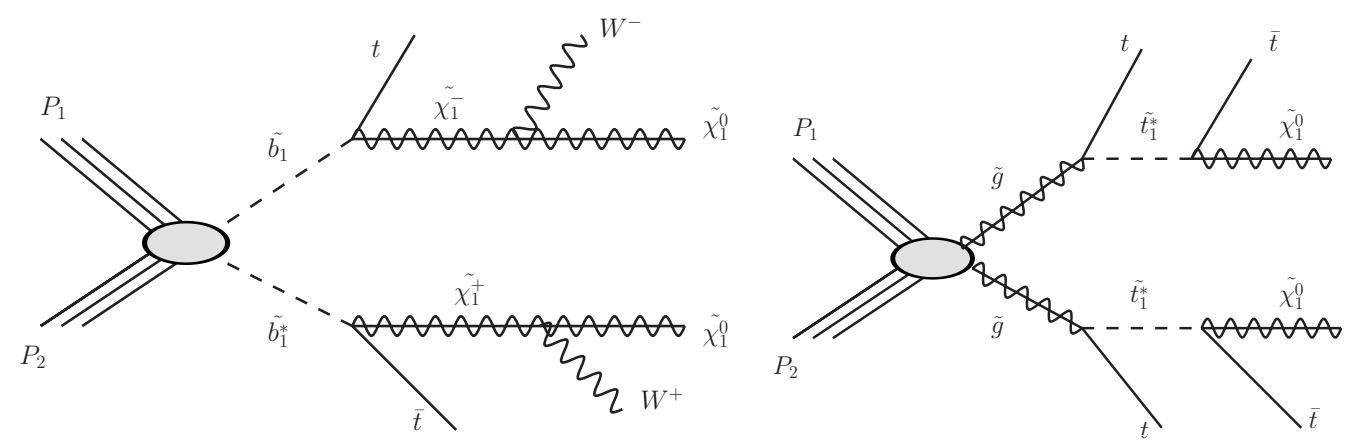

Figure 6: Direct pair production of sbottom quarks (left), and gluino-mediated production of stops (right), with decay chains which can lead to a signature with same-sign dileptons and at least 2 b-quark jets.
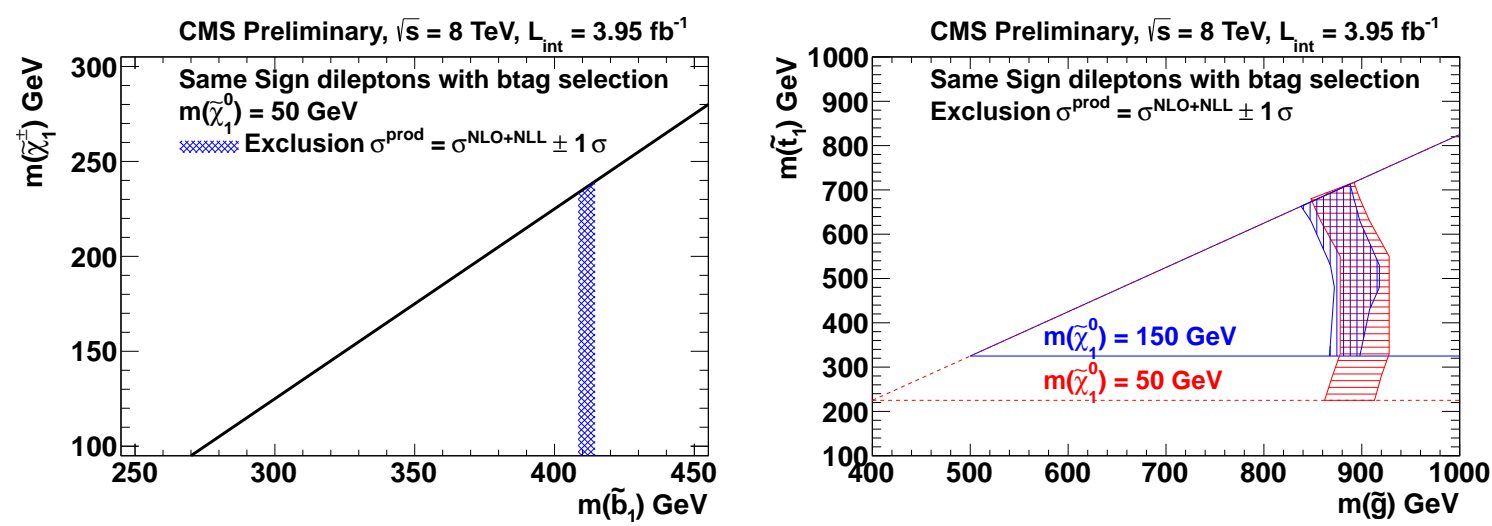

Figure 7: Exclusion (95 \% C.L.) in the (m(bottom), m(chargino)) plane for bottom squark pair production (left) and in the (m(stop),m(gluino)) plane for gluino decay to on-shell top squarks for different choices of the LSP mass (right)[32]. The lines represent the kinematic boundaries of the models. The regions to the left of the bands, and within the kinematic boundaries, are excluded; the thicknesses of the bands represent the theoretical uncertainties on the gluino pair production cross section from scale and parton distribution functions (pdf) variations.

chargino mass of $106 \mathrm{GeV}$, driven by LEP results, and equal to twice the neutralino mass. Once again, no constraint is produced for higher LSP masses, above $140 \mathrm{GeV}$. In addition, the region where $m(\tilde{t})+m(L S P) \sim m(t)$ is not excluded, allowing the possibility that the LSP is very light.

\section{Is SUSY hiding in the data?}

Even though no signal has been observed in the searches above, it is possible for SUSY to be present but undetected in the current data. Several regions of parameter space exist where small mass-splittings between the SUSY particles produce final states with low- $p_{T}$ objects, which can fall outside of the signal regions used. The unexplored band in Figure 8 is one example. In general, models with low mass splittings can be expected to produce signals with multiple jets and leptons as in classic RPC models, but less missing energy. The events therefore look more like the SM background. Initial state radiation may provide enough boost to the event to pass the kinematic selections, but the theoretical errors in estimating this effect are difficult to assess. ATLAS targeted 


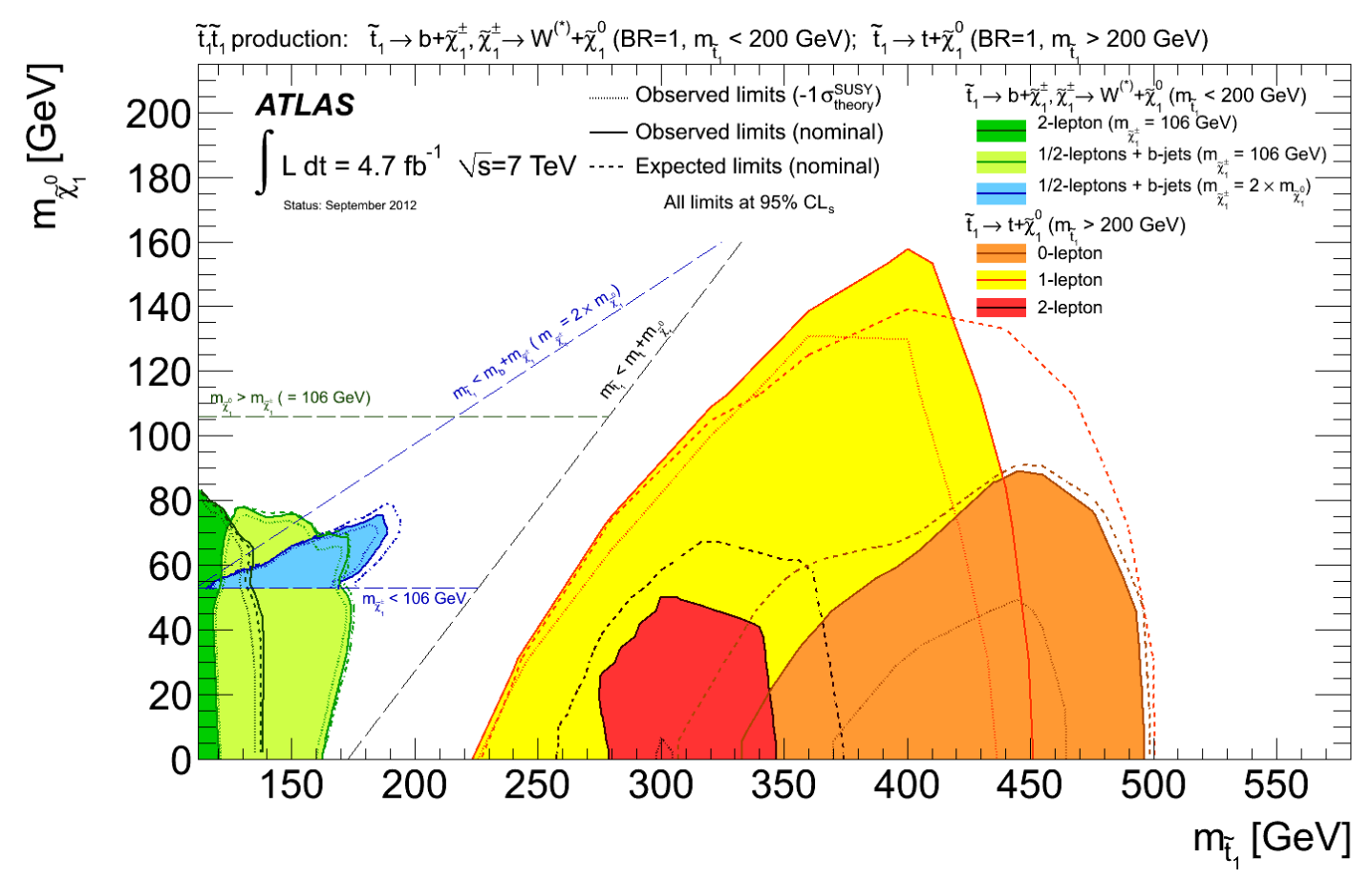

Figure 8: Summary of the five dedicated ATLAS searches for top squark (stop) pair production based on 4.7 $\mathrm{fb}^{-1}$ of pp collision data taken at $\sqrt{s}=7 \mathrm{TeV}$. Exclusion limits at 95\% CL are shown in the (stop,neutralino) mass plane. The dashed and solid lines show the expected and observed limits, respectively, including all uncertainties except the theoretical signal cross section uncertainty (PDF and scale). The dotted lines represent the results obtained when reducing the nominal signal cross section by $1 \sigma$ of its theoretical uncertainty. For stop masses below $200 \mathrm{GeV}$, the decay $\tilde{t}_{1} \rightarrow b+\tilde{\chi}_{1}^{ \pm}, \tilde{\chi}_{1}^{ \pm} \rightarrow W^{(*)}+\tilde{\chi}_{1}^{0}$ is assumed in all cases, with two hypotheses on the $\tilde{\chi}_{1}^{ \pm}, \tilde{\chi}_{1}^{0}$ mass hierarchy: $m\left(\tilde{\chi}_{1}^{ \pm}\right)=106 \mathrm{GeV}$ and $m\left(\tilde{\chi}_{1}^{ \pm}\right) \sim 2 m\left(\tilde{\chi}_{1}^{0}\right)$. For stop masses above $200 \mathrm{GeV}$, the decay $\tilde{t}_{1} \rightarrow t \tilde{\chi}_{1}^{0}$ is assumed to dominate.

this with a search for strong production of squarks and gluinos with small mass splittings [39], using low- $p_{T}$ leptons to reduce the background rate.

If masses are almost degenerate in the lightest part of the SUSY spectrum, it is possible that some particles are almost stable, and decays to the LSP occur outside of the beam-pipe, or even outside of the detector itself. ATLAS presented searches both for particle decays in flight [40], and for particles with very long lifetimes [41]. The first search was motivated by the degeneracy between the chargino and neutralino masses which occurs in anomaly-mediated symmetry breaking models. It focusses on decays inside the tracker volume, where the low- $p_{T}$ daughter track deviates from the path of the high- $p_{T}$ parent, leading to reconstructed tracks with very few hits in the ATLAS transition radiation tracker. The data shown in Figure 9 (left) excludes chargino masses below $118 \mathrm{GeV}$ for lifetimes between 1-2 ns. The second search, shown in Figure 9 (right), is motivated by almost stable heavy sleptons which can be detected by their longer time of flight to the outer detector. This data excludes stable sleptons with masses below $297 \mathrm{GeV}$, and staus in a gauge-mediated scenario with masses below $310 \mathrm{GeV}$.

R-parity is often assumed in SUSY models, not least because it offers the possibility of a stable LSP, which can account for dark matter. If R-parity is violated (RPV), then the LSP can ultimately 

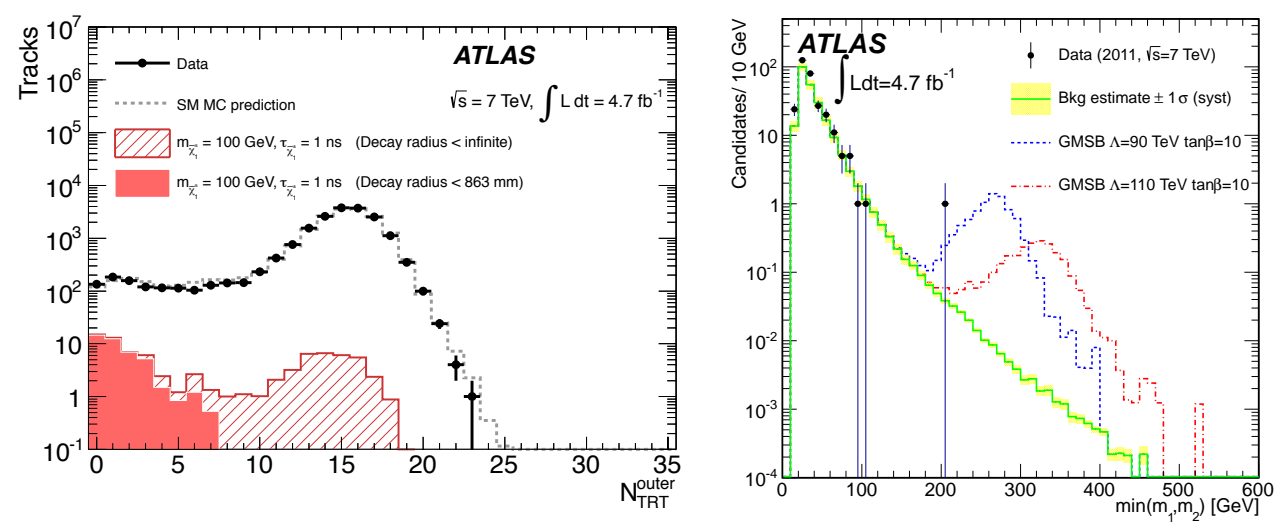

Figure 9: Left: The $\mathrm{N}_{T R T}^{\text {outer }}$ distribution for data and signal events $\left(\mathrm{m}_{\tilde{\chi}_{1}^{ \pm}}=100 \mathrm{GeV}, \tau_{\tilde{\chi}_{1}^{ \pm}}=1 \mathrm{~ns}\right)$ with the high- $\mathrm{p}_{T}$ isolated track selection. The expectation from SM MC events, normalized to the number of observed events, is also shown [40]. Right: Observed data, background estimate and expected signal in the two-candidate signal region in the slepton search [41]. Only the lower of the two masses is plotted.
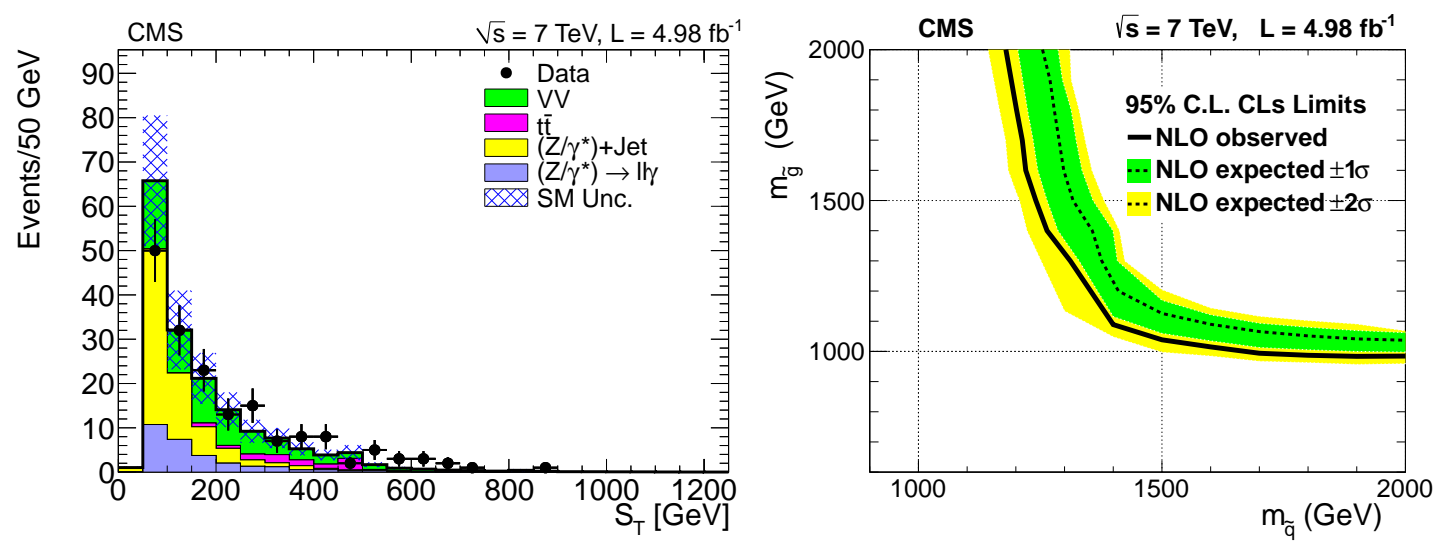

Figure 10: Left: $S_{T}$ data from the CMS multi lepton search, with 3 leptons, no $\tau$ s and a $\mathrm{Z}$ veto. Right: $95 \%$ $\mathrm{CL}_{s}$ exclusion limit for a scenario with leptonic RPV couplings, from [42].

decay to SM particles, and this can remove the missing energy signature used by many searches. CMS produced the first search for RPV scenarios using the $8 \mathrm{TeV}$ data [42]. This analysis used the relatively high production of leptons expected in SUSY models to reduce the SM background, as well as including missing energy and the presence of vector bosons and photons. A total of 53 channels were inspected, one of which is shown in Figure 10 (left), in this case requiring three leptons without any taus or $\mathrm{Z}$ bosons. The $S_{T}$ discriminant is a scalar sum of the energy of the jets, the $p_{T}$ of the leptons and the missing transverse energy. Figure 10 (right) shows one of the many exclusion limits derived from the data, for the purely leptonic RPV coupling $\lambda_{e \mu \tau}$.

While RPV offers one way to evade the current search bounds, another possibility is that the SUSY phenomenology is altered by an extension to the minimal framework, for example by additional light hidden sector particles as proposed in the "stealth" scenario of [43]. A possible decay chain is shown in Figure 11 (left), which produces events with diphotons, mediated by the 

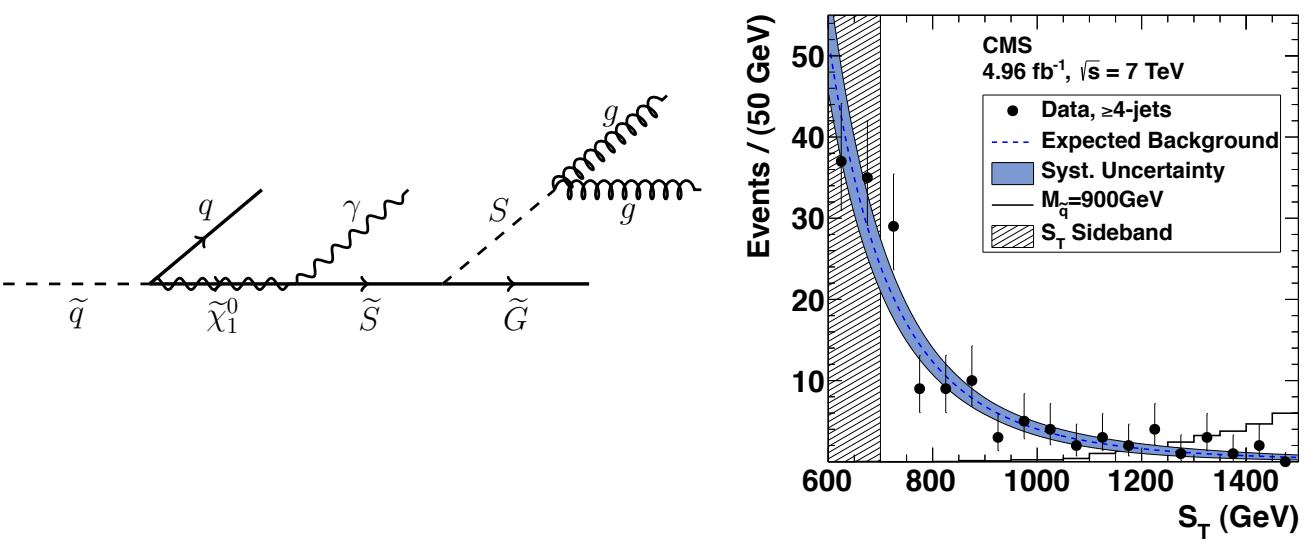

Figure 11: Left: Decay of a squark in stealth SUSY [43]. Right: Observed $S_{T}$ spectrum, background expectation with systematic uncertainty, and predicted signal for a squark mass of $900 \mathrm{GeV}$ in events with four or more jets [44].

hidden sector "singlinos". CMS presented a novel search for this scenario [44], with one result shown in Figure 11 (right). The signal is expected at high values of the total transverse energy $S_{T}$.

\section{Conclusions: is SUSY dead?}

The data are now putting pressure on SUSY models from many directions, but it would be completely premature to suggest that the idea is dead. Some of the simplest and more constrained models, such as the MSSM, find it difficult to remain compatible with the data without implausible levels of fine-tuning. However, all SUSY scenarios need to make simplifying assumptions, and care must therefore be taken when applying the exclusion plots to particular models. There are many "natural" SUSY scenarios which are not ruled out: models with light stops or RPV still have few constraints. We can therefore look forward with hope. SUSY remains the best motivated extension to the standard model, and the searches are now so powerful and extensive that they should find any trace of its existence in the LHC energy range. With the increasing energy and luminosity of this magnificent machine, the experiments will be busy with this work for some years to come.

\section{References}

[1] H. Miyazawa, Baryon Number Changing Currents, Prog. Theor. Phys. 36 (6) (1966) 1266.

[2] P. Ramond, Dual Theory for Free Fermions, Phys. Rev. D 3 (1971) 2415.

[3] Y. A. Golfand and E. P. Likhtman, Extension of the Algebra of Poincare Group Generators and Violation of p Invariance, JETP Lett. 13 (1971) 323. [Pisma Zh. Eksp. Teor. Fiz. 13 (1971) 452-455].

[4] A. Neveu and J. H. Schwarz, Factorizable dual model of pions, Nucl. Phys. B 31 (1971) 86.

[5] A. Neveu and J. H. Schwarz, Quark Model of Dual Pions, Phys. Rev. D 4 (1971) 1109.

[6] J. Gervais and B. Sakita, Field theory interpretation of supergauges in dual models, Nucl. Phys. B 34 (1971) 632. 
[7] D. V. Volkov and V. P. Akulov, Is the Neutrino a Goldstone Particle?, Phys. Lett. B 46 (1973) 109.

[8] J. Wess and B. Zumino, A Lagrangian Model Invariant Under Supergauge Transformations, Phys. Lett. B 49 (1974) 52.

[9] J. Wess and B. Zumino, Supergauge Transformations in Four-Dimensions, Nucl. Phys. B 70 (1974) 39.

[10] ATLAS Collaboration, The ATLAS Experiment at the CERN Large Hadron Collider, JINST 3 (2008) S08003.

[11] CMS Collaboration, The CMS experiment at the CERN LHC, JINST 3 (2008) S08004.

[12] H. Goldberg, Constraint on the photino mass from cosmology, Phys. Rev. Lett. 50 (1983) 1419.

[13] J. Ellis, J. Hagelin, D. Nanopoulos, K. Olive, and M. Srednicki, Supersymmetric relics from the big bang, Nucl. Phys. B238 (1984) 453-476.

[14] S. Dimopoulos and H. Georgi, Softly Broken Supersymmetry and SU(5), Nucl. Phys. B 193 (1981) 150.

[15] E. Witten, Dynamical Breaking of Supersymmetry, Nucl. Phys. B188 (1981) 513.

[16] M. Dine, W. Fischler, and M. Srednicki, Supersymmetric Technicolor, Nucl. Phys. B189 (1981) 575-593.

[17] S. Dimopoulos and S. Raby, Supercolor, Nucl. Phys. B192 (1981) 353.

[18] N. Sakai, Naturalness in Supersymmetric Guts, Zeit. Phys. C11 (1981) 153.

[19] R. Kaul and P. Majumdar, Cancellation of quadratically divergent mass corrections in globally supersymmetric spontanneously broken gauge theories, Nucl. Phys. B199 (1982) 36.

[20] ATLAS Collaboration, Search for squarks and gluinos with the ATLAS detector in final states with jets and missing transverse momentum using $4.7 \mathrm{fb}^{-1}$ of $\sqrt{s}=7 \mathrm{TeV}$ proton-proton collision data, arXiv:1208.0949 [hep-ex].

[21] CMS Collaboration, Search for new physics in the multijet and missing transverse momentum final state in proton-proton collisions at $\sqrt{s}=7 \mathrm{TeV}$, Phys. Rev. Lett. 109 (2012) 171803 [arXiv:1207.1898 [hep-ex]].

[22] CMS Collaboration, CMS-PAS-SUS-12-016, Search for supersymmetery in final states with missing transverse momentum and 0, 1, 2, or $\geq 3$ b jets in $8 \mathrm{TeV}$ pp collisions, 2012, [https://cdsweb.cern.ch/record/1460095]

[23] CMS Collaboration, Search for new physics in events with opposite-sign leptons, jets, and missing transverse energy in pp collisions at sqrt(s) = $7 \mathrm{TeV}$, arXiv:1206.3949 [hep-ex].

[24] ATLAS Collaboration, Search for direct slepton and gaugino production in final states with two leptons and missing transverse momentum with the ATLAS detector in pp collisions at $\sqrt{s}=7 \mathrm{TeV}$, arXiv:1208.2884 [hep-ex].

[25] LEP SUSY Working Group (ALEPH, DELPHI, L3, OPAL), Notes LEPSUSYWG/01-03.1 and 04-01.1, http://lepsusy.web.cern.ch/lepsusy/Welcome.html.

[26] P. Fayet, Supersymmetry and Weak, Electromagnetic and Strong Interactions, Phys. Lett. B64 (1976) 159.

[27] P. Fayet, Spontaneously Broken Supersymmetric Theories of Weak, Electromagnetic and Strong Interactions, Phys. Lett. B69 (1977) 489. 
[28] G. R. Farrar and P. Fayet, Phenomenology of the Production, Decay, and Detection of New Hadronic States Associated with Supersymmetry, Phys. Lett. B76 (1978) 575-579.

[29] P. Fayet, Relations Between the Masses of the Superpartners of Leptons and Quarks, the Goldstino Couplings and the Neutral Currents, Phys. Lett. B84 (1979) 416.

[30] R. Barbieri and G. Giudice, Upper Bounds on Supersymmetric Particle Masses, Nucl.Phys. B306 (1988) 63.

[31] B. de Carlos and J. Casas, One loop analysis of the electroweak breaking in supersymmetric models and the fine tuning problem, Phys.Lett. B309 (1993) 320-328, arXiv: hep-ph/9303291 [hep-ph].

[32] CMS Collaboration, CMS-PAS-SUS-12-017, Search for supersymmetry in events with same-sign dileptons, 2012, [http://cdsweb.cern.ch/record/1459811]

[33] CMS Collaboration, CMS-PAS-SUS-12-004, Search for supersymmetry in all-hadronic events with tau leptons, 2012, [http://cdsweb.cern.ch/record/1459809]

[34] ATLAS Collaboration, Search for light scalar top quark pair production in final states with two leptons with the ATLAS detector in $\sqrt{s}=7$ TeV proton-proton collisions, arXiv:1208.4305 [hep-ex].

[35] ATLAS Collaboration, Search for light top squark pair production in final states with leptons and $b^{-}$ jets with the ATLAS detector in $\sqrt{s}=7 \mathrm{TeV}$ proton-proton collisions, arXiv:1209.2102 [hep-ex].

[36] ATLAS Collaboration, Search for a supersymmetric partner to the top quark in final states with jets and missing transverse momentum at $\sqrt{s}=7 \mathrm{TeV}$ with the ATLAS detector, arXiv:1208.1447 [hep-ex].

[37] ATLAS Collaboration, Search for direct top squark pair production in final states with one isolated lepton, jets, and missing transverse momentum in $\sqrt{s}=7 \mathrm{TeV}$ pp collisions using 4.7 fb-1 of ATLAS data, arXiv:1208.2590 [hep-ex].

[38] ATLAS Collaboration, Search for a heavy top-quark partner in final states with two leptons with the ATLAS detector at the LHC, arXiv:1209.4186 [hep-ex].

[39] ATLAS Collaboration, Further search for supersymmetry at $\sqrt{s}=7 \mathrm{TeV}$ in final states with jets, missing transverse momentum and isolated leptons with the ATLAS detector, Phys. Rev. D 86 (2012) 092002 [arXiv:1208.4688 [hep-ex]].

[40] ATLAS Collaboration, Search for direct chargino production in anomaly-mediated supersymmetry breaking models based on a disappearing-track signature in pp collisions at $\sqrt{s}=7$ TeV with the ATLAS detector, arXiv:1210.2852 [hep-ex].

[41] ATLAS Collaboration, Searches for heavy long-lived sleptons and R-Hadrons with the ATLAS detector in pp collisions at sqrt(s) = $7 \mathrm{TeV}$, arXiv:1211.1597 [hep-ex].

[42] CMS Collaboration, Search for anomalous production of multilepton events in pp collisions at $\operatorname{sqrt}(s)=7$ TeV, JHEP 1206 (2012) 169 [arXiv:1204.5341 [hep-ex]].

[43] J. Fan, M. Reece and J. T. Ruderman, Stealth Supersymmetry, JHEP 1111 (2011) 012 [arXiv:1105.5135 [hep-ph]].

[44] CMS Collaboration, Search for supersymmetry in events with photons and low missing transverse energy in pp collisions at $\sqrt{s}=7 \mathrm{TeV}$, Submitted to: Phys. Lett. B. [arXiv:1210.2052 [hep-ex]]. 